\title{
Mathematical Modeling and Experimental Validation of an Electric Arc Furnace
}

\author{
Vito LOGAR, Dejan DOVŽAN and Igor ŠKRJANC \\ Laboratory of Modeling, Simulation and Control Faculty of Electrical Engineering, University of Ljubljana, Tržaška 25, SI-1000 \\ Ljubljana, Slovenia. E-mail: vito.logar@fe.uni-lj.si
}

(Received on September 30, 2010; accepted on November 4, 2010)

\begin{abstract}
The following paper presents an approach to the mathematical modeling of 3-phase $A C$, electric arc furnace (EAF) processes for control-design and process-optimization purposes. The EAF can be, from the modeling point of view, considered as a combination of electrical, hydraulic, chemical, thermal and several energy-balance sub-processes or sub-models. In this paper the modeling of the electrical and hydraulic submodels is presented in detail, since the two represent a very complex and important sub-system of the complete EAF model. The presented sub-models are obtained in accordance with different mathematical, electrical and mechanical laws. Several parameters, which are necessary to successfully identify the scrapmelting process, were fitted experimentally, using the measured operational data of an 80 MVA AC furnace during different periods of the melting process. Similar data has also been used for the validation of the developed model in typical EAF operating situations. The aim of the presented EAF modeling is to obtain an accurate, robust and realistic mathematical model of the scrap-melting process, which will later be used for control-design purposes, the optimization of the energy consumption and the development of an operatortraining simulator. The main advantage of our modeling approach over the existent EAF-related models is a more macroscopic level of modeling, which accurately simulates the electrical and hydraulic processes under different conditions in the EAF.
\end{abstract}

KEY WORDS: EAF; electric model; hydraulic model; experimental validation; harmonic analysis.

\section{Introduction}

In this paper an approach to the mathematical modeling of the 3-phase AC, electric arc furnace (EAF) scrap-melting process and operation is presented. Today, EAF-recycled scrap metal represents $1 / 3$ of the world's annual steel production. Since a typical EAF consumes approximately $450 \mathrm{kWh}$ /ton of electrical energy during its operation, the EAF and its processes represent an interesting research field from the electrical, economic and ecological points of view.

There are already a number of papers that present various modeling approaches to several sub-systems of electric arc furnaces, from basic arc models ${ }^{1,2)}$ to complex flicker simulations, ${ }^{3-7)}$ which tend to prevail due to the enormous impact of EAFs on electrical networks. Since the EAF represents a complex system these articles usually focus on a certain EAF-related problem and in some instances use simulated, laboratory-generated data for the analysis, which is easier to process and gives more convenient results. However, in this paper the process of EAF scrap refining is modeled on a more macroscopic level, using measurements from an actual EAF operation, trying to build a model that accurately simulates the electrical and hydraulic processes under different conditions and during different melting periods in the furnace. Therefore, special attention is devoted to the development and parametrization of the model in order to precisely represent the measured data, which will then be used for control-design purposes, energy-consumption optimization and the development of an operator-training simulator.

The process of scrap refining can be divided into several phases, from sorting the metal, loading the baskets, melting, to tapping and secondary metallurgy, etc. This paper looks at the melting period of the steel production, as it probably represents the most complex and the most important phase of the overall refining process. The principle of melting the scrap in an EAF is to transform the electrical energy into thermal energy, which is dissipated from the electric arcs, to melt the materials loaded in the furnace. The arcs burn between the graphite electrodes and the conducting scrap (3-phase, no neutral), and are characterized by low voltages ( $\sim 400-1000 \mathrm{~V}$ per phase) and high currents $(\sim 40-60 \mathrm{kA}$ per phase). The temperatures in the arc's core can reach up to $8500 \mathrm{~K}$, at which point the gas discharge holds an electrical conductivity of $\sim 10^{3} \mathrm{~S} / \mathrm{m}$. In this way the scrap can be melted reasonably quickly and efficiently.

The model of the EAF presented in this work is based on the 80 MVA AC furnace installed in one of the ironworks in Slovenia. For this reason, operational data relating to the EAF in different operating situations has been collected, with the measurements including several electrical (powers; voltages; currents; power factors; operating resistances and 
reactances; etc.) and non-electrical (transformer and reactor taps; number of short circuits and arc breakages; electrode controller outputs; electrode position; temperatures of the cooling panels; composition and weight of the additives; consumption of oxygen, carbon, gas; etc.) values sampled over a 1-s time window. At this stage of the model's development, nearly all of the electrical and some of the mechanical values were used to obtain the correct parameters of the sub-models describing the electrical properties of the EAF. Additionally, since the properties of the electric arcs are dependent on the position of the hydraulically actuated electrodes, the presented hydraulic sub-model for the electrode control completes the particular modeling assembly.

\section{Measurements and Modeling}

\subsection{EAF Operation Data}

For the purposes of this study, the measurements were made during different operational situations in the EAF melting process. The obtained data included measurements of the effective values, i.e., the root mean squares (RMS), sampled over a 1-s window of the following: phase voltages; phase-to-phase voltages; arc voltages; phase currents; power factors; arc resistances and reactances; total circuit resistances and reactances; apparent, active, reactive and arc power; total energy consumption; etc. The measurements were made with the electrode-control and data-acquisition system (E.M.P.E.R.E.). ${ }^{8)}$

\subsection{Modeling}

The following section presents the approach to modeling the electrical circuits and electrode control of the particular EAF. There are many possible methods and tools that can be used for developing the EAF mathematical model; nonetheless, in this study the authors decided to use harmonic analysis in combination with a discrete Matlab simulation (fixed step, $T_{\mathrm{s}}=10^{-4} \mathrm{~s}$ ), which proved to be efficient and less time consuming than other possible modeling/simulation approaches (Matlab-Simulink-Simscape, etc.). Therefore, the secondary transformer voltages, which are the only electrical input to the model can be described by Eq. (1):

$$
\begin{aligned}
& U_{1}=U_{\text {tap }} \cdot e^{j 2 \pi f t} \\
& U_{2}=U_{\text {tap }} \cdot e^{j 2 \pi f t+(2 \pi / 3)} \\
& U_{3}=U_{\text {tap }} \cdot e^{j 2 \pi f t+(4 \pi / 3)}
\end{aligned}
$$

where $U_{\text {tap }}$ denotes the transformer voltage amplitude (chosen by the transformer tap: $600-981 \mathrm{~V}$ in 18 taps), $f$ represents the network frequency $(50 \mathrm{~Hz})$ and $t$ represents the time. In this manner, all the dependent electrical values inherit the complex characteristics.

\subsubsection{Electric Circuits}

One of the main EAF systems is represented by the furnace transformer, connected to the HV/MV 110-35 kV distribution station via the 19.3 MVA furnace reactor (reactive load). The rated power of the transformer is $80 \mathrm{MVA}$, with the secondary side voltage range from 600 to $980 \mathrm{~V}$, divided into 18 steps (transformer taps). The rated current on the

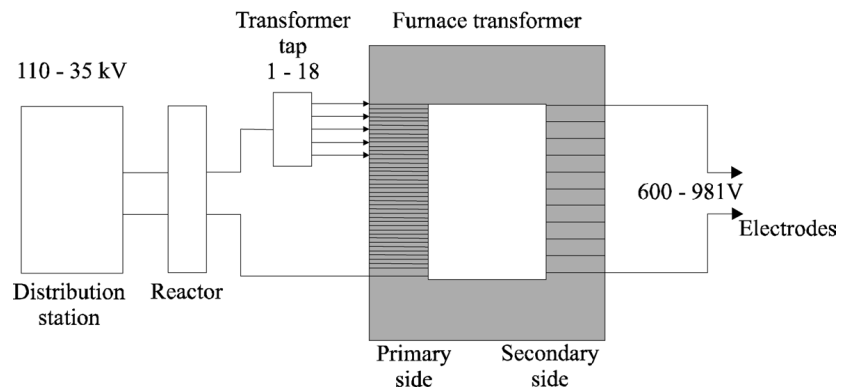

Fig. 1. Schematic representation of the 80 MVA EAF electric circuit.

primary side extends from 1.060 to $1.320 \mathrm{~A}$ and from 47 to $62 \mathrm{kA}$ on the secondary side. The allowable 2-s short-circuit currents range up to $16 \mathrm{kA}$ on the primary side and up to $900 \mathrm{kA}$ on the secondary side. A simplified EAF electrical scheme is shown in Fig. 1.

The furnace reactor represents a variable reactive (inductive) load in the range from 3.89 to $0 \mathrm{~m} \Omega$ divided into 6 equidistant steps (reactor taps). The reactive load added is primarily used in the initial phases of the meltdown process to increase the circuit's reactance and therefore, also the stability of the arcs, which are highly unstable and often extinguish due to scrap in solid form and the chaotic conditions in the furnace.

The electrical model of the EAF can be described as a 3phase, non-linear electric circuit, where the electric arcs represent the main non-linearities. Mathematically, the electric arc can be described as an impedance load, whose resistance $R_{\text {arc }}$ is modeled by means of the Cassie-Mayr model $^{2,9,10)}$ from Eq. (2). Due to possible numerical problems, the arc resistance $R_{\text {arc }}$ is expressed in a logarithmic form $s$ :

$$
\frac{d s}{d t}=\frac{1}{\tau} \cdot\left(1-\frac{U_{\mathrm{arc}} I}{P_{0}} \cdot e^{\alpha s}\right), \quad s=\ln R_{\mathrm{arc}}
$$

where $R_{\text {arc }}$ represents the arc resistance, $U_{\text {arc }}$ represents the arc voltage, $I$ represents the phase current, $\alpha$ represents the Cassie-Mayr constant ( $\alpha=0-$ Mayr model for low current values, $\alpha=1-$ Cassie model for high current values), $\tau$ represents the cooling constant of the arc (set experimentally to $\sim 7 \cdot 10^{-4} \mathrm{~s}$ ) and $P_{0}$ represents the arc's power dissipation, described by Eq. (3):

$$
P_{0}=2 \pi^{1 / 2} \sigma^{-1 / 2} l^{3 / 2} \sigma_{\mathrm{SB}} T^{4}
$$

where $\sigma$ represents the specific conductivity of the ionized air $\left(2 \cdot 10^{3} \mathrm{~S} / \mathrm{m}\right), \sigma_{\mathrm{SB}}$ represents the Stefan-Boltzmann constant $\left(5.6704 \cdot 10^{-8} \mathrm{~J} \mathrm{~s}^{-1} \mathrm{~m}^{-2} \mathrm{~K}^{-4}\right), l$ represents the arc length and $T$ represents the mean temperature of the arc pillar (set experimentally to $\sim 4.500 \mathrm{~K}$ ). Equation (3) and the time constant $\tau$ in Eq. (2) define the time that is needed to heat the arc to achieve its steady state $U_{\text {arc }} I=P_{0}{ }^{2}$ )

Furthermore, the arc's reactance $X_{\text {arc }}$ is in the literature modeled in a few possible ways, i.e., ${ }^{11-13)}$ which all yield similar results when applied in practice. In this study we expressed the operational reactance of the $\operatorname{arcs} X_{\text {arc }}$ by means of the Köhle model ${ }^{13)}$ in Eq. (4): 


$$
\frac{X_{\mathrm{arc}}}{X_{0}}=K_{1} \cdot \frac{R_{\mathrm{arc}}}{X_{0}}+K_{e} \cdot\left(K_{2} \cdot \frac{R_{\mathrm{arc}}}{X_{0}}+K_{3} \cdot \frac{R_{\mathrm{arc}}^{2}}{X_{0}^{2}}\right)
$$

where $X_{0}$ represents the EAF short-circuit reactance, the coefficients $K_{1}, K_{2}$ and $K_{3}$ represent the dependence of $X_{\text {arc }}$ from the resistance $R_{\text {arc }}$, while the parameter $K_{e}$, can be described by Eq. (5):

$$
K_{e}=e^{-\left(t / T_{x}\right)}
$$

and describes the exponential decrease of the reactance with time. $T_{x}$ represents a time constant, which is set experimentally to $20 \mathrm{~min}$. The exponential impedance drop is a consequence of the scrap-melting and slag-forming process, i.e., in the initial stage of meltdown, with a lack of molten steel and slag, the arc's impedance load is high (due to the distortion of the arc's current and the voltage sineshape and the high instability of the arc burning), while it gradually decreases to a minimum value at the end of the melting period (all steel molten-flat bath). The values of the parameters $K_{1}$ to $K_{3}$ were set to $K_{1}=0.1, K_{2}=0.15$ and $K_{3}=0.05$ and were fitted experimentally to achieve the most realistic match between the measured and simulated reactance decrease. The (virtual) increase in the arc's reactance is, together with other constant reactances, referred to as the operational inductive resistance or the operational reactance of the EAF.

According to Eqs. (2) and (4) the arc impedance can be obtained by Eq. (6):

$$
Z_{\mathrm{arc}}=\sqrt{R_{\mathrm{arc}}^{2}+X_{\mathrm{arc}}^{2}}
$$

which is a well-known equation for calculating the load's impedance.

According to the literature, ${ }^{14,15)}$ the arc voltages are reduced by about $30-40 \mathrm{~V}$ due to the cathode voltage drop, which can presumably be represented as an additional resistance. Therefore, observing Fig. 2, the actual arc impedance $Z_{\text {arc }}$ is by $R_{\text {cathode }}$ smaller than the $Z_{\text {arc }}$ calculated in Eq. (7):

$$
Z_{\text {arc }}=Z_{\text {arc }}-R_{\text {cathode }}=Z_{\text {arc }}-\frac{U_{\text {cathode }}}{I}
$$

where $U_{\text {cathode }}$ is the corresponding cathode voltage drop.

The EAF electric-circuit model, including the transformer impedances, the line impedances, the arc impedances and the additional media impedances (i.e., scrap, slag impedance etc.) can be represented as a three-phase, star AC circuit, shown in Fig. 2.

In order to successfully develop an electric model of the EAF all the impedances in Fig. 2 have to be known. While the media impedance has to be fitted experimentally and the arc impedance is given by Eq. (7), two other impedances $\left(Z_{\text {tr }}\right.$ and $\left.Z_{\text {line }}\right)$ are given or can be computed. The transformer and the line resistances are known and add up to $R_{\mathrm{tr}}=0.37 \mathrm{~m} \Omega$ and $R_{\text {line }}=0.12 \mathrm{~m} \Omega$. The line reactances are also known and add up to $X_{\text {line }}=1.08 \mathrm{~m} \Omega$. On the other hand, the inner transformer reactance and the reactor reactance reflected over the transformer core have to be computed by means of Eq. (8):

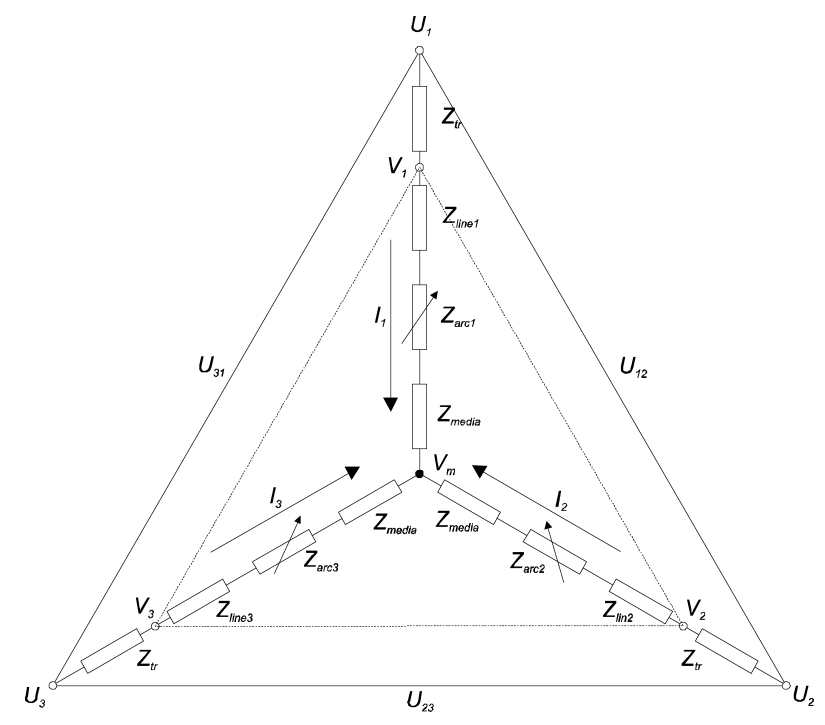

Fig. 2. Electric diagram $/$ model of an EAF; $U_{1}, U_{2}, U_{3}$, phase voltages; $U_{12}, U_{23}, U_{31}$, phase-to-phase voltages; $V_{1}, V_{2}$, $V_{3}$, phase voltages without transformer-voltage drop; $I_{1}$, $I_{2}, I_{3}$, phase currents; $Z_{\mathrm{tr}}$, transformer and reactor impedance; $Z_{\text {line }}$, line impedances; $Z_{\text {arc }}$, arc impedances; $Z_{\text {media }}$, media impedance; $V_{\mathrm{m}}$, neutral point potential.

$$
\begin{gathered}
X_{\text {transformer }}=\frac{u_{k}}{100 \%} \cdot \frac{U_{\text {tap }}^{2}}{80 \mathrm{MVA}} \\
X_{\text {reactor }}=X_{\text {rtap }} \cdot\left(\frac{U_{\text {tap }}}{35 \mathrm{kV}}\right)^{2} \cdots \\
X_{\text {tr }}=X_{\text {transformer }}+X_{\text {reactor }}
\end{gathered}
$$

where $X_{\text {transformer }}$ represents the transformer reactance, $X_{\text {reactor }}$ represents the reactor reactance, $X_{\text {tr }}$ represents the inner reactance used for further computation, $u_{k}$ represents the impedance voltage according to the selected transformer tap in $\%$ (from 16.6 to $6.3 \%$ in 18 transformer taps), $U_{\text {tap }}$ represents the secondary transformer voltage according to the selected transformer tap, $X_{\text {rtap }}$ represents the rated reactor impedance according to the selected reactor tap (from 3.89 to $0 \mathrm{~m} \Omega$ in 6 reactor taps), and $80 \mathrm{MVA}$ and $35 \mathrm{kV}$ represent the transformer's rated power and primary side voltage, respectively. ${ }^{16)}$

From the Eqs. (2) and (4) it is obvious that the impedances of the electric arcs represent non-linear, time-variant loads, which consequently causes an unbalanced furnace operation. Therefore, the three-phase neutral point $V_{\mathrm{m}}$, which has a potential of $0 \mathrm{~V}$ when operating with balanced/symmetrical furnace loads in all three phases, moves inside the triangle bounded by the voltages $U_{12}, U_{23}, U_{31}$; typically, in a circle, whose diameter is proportional to the arc's impedance $Z_{a}$. The position of the potential on the circle is defined by the phase shift of the neutral point potential $V_{\mathrm{m}}$ to the phase-to-phase voltages. $V_{\mathrm{m}}$ reaching the edge of a triangle is a sign that one of the arcs is extinguished and needs to be re-ignited.

Mathematically, the neutral point potential $V_{\mathrm{m}}$ can be determined by Eq. (9): 


$$
V_{\mathrm{m}}=\frac{\sum_{i=1}^{3} Y_{i} \cdot U_{i}}{\sum_{i=1}^{3} Y_{i}}
$$

where $Y_{i}$ represents the sum of the admittances in each phase and $U_{i}$ represents the phase voltages.

The voltages and phase currents can be computed by means of the well-known Kirchhoff's voltage and current laws. The total voltage drop on the transmission lines, arc and media for each phase $(i=1, \cdots, 3)$ can be computed from Eq. (10):

$$
V_{i}=\frac{\left(Z_{\text {arc } \_i}+Z_{\text {line } \_i}+Z_{\text {media }}\right) \cdot\left(U_{i}-V_{\mathrm{m}}\right)}{Z_{\text {arc } \_i}+Z_{\text {line } \_i}+Z_{\text {media }}+Z_{\text {tr }}}
$$

and the phase currents can be obtained from Eq. (11):

$$
I_{i}=\frac{U_{i}-V_{\mathrm{m}}}{Z_{\text {arc } \_i}+Z_{\text {line } \_i}+Z_{\text {media }}+Z_{\text {tr }}}
$$

The arc's voltage drop can be computed by means of the Eq. (12):

$$
V_{\text {arc } \_i}=I_{i} \cdot Z_{\text {arc } \_i}-V_{\text {cathode }}=I_{i} \cdot Z_{\text {arc } \_i}-I_{i} \cdot R_{\text {cathode }}
$$

The total apparent $(S)$, active $(P)$, arc $\left(P_{\text {arc }}\right)$ and reactive $(Q)$ powers of the EAF can be obtained by combining the voltages and the currents computed in Eqs. (10), (11) and (12) by Eqs. (13), (14), (15) and (16):

$$
\begin{aligned}
& S=\sum_{i=1}^{3} S_{i}=\sum_{i=1}^{3} I_{i}^{2} \cdot\left(Z_{\text {arc } \_i}+Z_{\text {line } \_i}+Z_{\text {media }}+Z_{\text {tr }}\right) \\
& P=\sum_{i=1}^{3} P_{i}=\sum_{i=1}^{3} I_{i}^{2} \cdot\left(R_{\text {arc } \_i}+R_{\text {line } \_i}+R_{\text {media }}+R_{\mathrm{tr}}\right) \\
& P_{\mathrm{arc}}=\sum_{i=1}^{3} P_{\mathrm{arc} \_i}=\sum_{i=1}^{3} I_{i}^{2} \cdot R_{\mathrm{arc} \_i} \\
& Q=\sum_{i=1}^{3} Q_{i}=\sum_{i=1}^{3} \sqrt{S_{i}^{2}-P_{i}^{2}}
\end{aligned}
$$

The power factors can be obtained by Eq. (17):

$$
\cos \varphi_{i}=\frac{P_{i}}{S_{i}}
$$

In order to successfully identify the developed mathematical model and to achieve the optimal match between the measured and the simulated data, appropriate values for the rest of the required parameters should be obtained: $Z_{\text {media }}$, media impedance $(\sim 0.45 \mathrm{~m} \Omega) ; R_{\text {cathode }}$, factor of cathode voltage drop $(\sim 0.8 \mathrm{~m} \Omega)$. The parameters were fitted experimentally, observing the real and simulated data and altering their values to obtain a satisfactory response for each sub-model. Since the conditions in a furnace change according to the selected transformer and reactor taps, the values of the mentioned parameters are not constant but change along with the selected taps.

\subsubsection{Chaos}

Since the process of melting exhibits excessive chaotic behavior, which cannot be described by conventional electric laws, a form of randomness has been added to the existing mathematical equations to achieve a greater similarity between the measured and simulated data. There are a few possible ways to do so, i.e., random noise, Lorentz attractor, etc. ${ }^{17)}$ The model proposed in this paper was simulated using both options, however, slightly better results were achieved when using the Lorentz attractor. This Lorentz attractor can be described by Eq. (18):

$$
\begin{aligned}
& \frac{d x_{i}}{d t}=K_{L} \cdot \sigma_{L} \cdot\left(y_{i}-x_{i}\right) \\
& \frac{d y_{i}}{d t}=K_{L} \cdot x_{i} \cdot\left(\rho_{L}-z_{i}\right)-K_{L} \cdot y_{i} \\
& \frac{d z_{i}}{d t}=K_{L} \cdot x_{i} \cdot y_{i}-K_{L} \cdot \beta_{L} \cdot z_{i}
\end{aligned}
$$

where $K_{L}$ denotes the frequency of the attractor (set to $\left.K_{L}=55\right), \sigma_{L}, \rho_{L}$ and $\beta_{L}$ represent the attractor parameters (set to $\sigma_{L}=10, \rho_{L}=58$ and $\beta_{L}=8 / 3$ ) and $x_{i}, y_{i}, z_{i}$ represent the attractor states for each of the arcs separately. Therefore, the resistance of the arcs is altered by adding the randomness of the attractor, as described by Eq. (19):

$$
R_{\text {arc } \_i}=R_{\text {arc } \_i}+\left(x_{i}+y_{i}+z_{i}\right) \cdot K_{t}-\overline{\left(x_{i}+y_{i}+z_{i}\right)} \cdot K_{t} \ldots
$$

where $K_{t}$ represents the time-variant attractor gain, and $\overline{\left(x_{i}+y_{i}+z_{i}\right)}$ represents the mean value of the attractor's states (to retain the average value of the $R_{\text {arc } \_i}$ ). The gain factor $K_{t}$ is larger at the beginning of the melting process, as it is known that the initial periods express higher degrees of chaotic behavior than the final periods, due to the lower temperatures, the solid steel and the lack of slag. The experimental results showed that an optimal chaotic match between the measured and simulated data can be obtained when $K_{t}$ is described with the following non-linear equation:

$$
K_{t}=\left(e^{-\left(t_{\text {tap }} / T_{t 1}\right)} \cdot \frac{2}{3}+\frac{1}{3}\right) \cdot\left(e^{-\left(t / T_{t 2}\right)} \cdot \frac{1}{2}+\frac{1}{2}\right) \ldots \ldots
$$

where $t_{\text {tap }}$ represents the time measured from the beginning of each basket loading, $T_{t 1}$ and $T_{t 2}$ represent the exponential decrease time constants (for the case of our study experimentally set to $200 \mathrm{~s}$ and $1600 \mathrm{~s}$ respectively) and $t$ represents the time measured from the beginning of the melting process.

\subsubsection{Electrode Control}

As already mentioned, the aim of the EAF modeling is to develop a reliable mathematical model, which shall mainly be used for the optimization of the energy consumption ( similar to MacRosty ${ }^{18)}$ ). Since the electrode control represents one of the crucial sub-systems for proper EAF operation, obtaining a realistic and reliable model for the electrode control is significant. A hydraulically actuated electrode-control system can be considered as one of the few control-related problems of the EAF and represents an im- 
portant part of the furnace operation, since the position of the electrodes directly influences the length of the arcs and consequently their impedances. The length of each arc, together with the current EAF settings (transformer tap, reactor tap) affects the arc's power. Therefore, the purpose of the electrode-positioning system is to control the resistance (through the length) and the power of each arc according to the selected operation program.

Although the dynamics and problems of the hydraulic systems are well known, thus far, a mathematical model for an EAF electrode-positioning system cannot be found in existing publications. ${ }^{19,20)}$

The hydraulic electrode-control system is presented schematically in Fig. 3.

As Fig. 3 demonstrates, the hydraulic circuit roughly consists of a hydraulic tank, pumps, cylinders, a secondary supply and the control valves. The controller of electrodes operates three control valves, which adjust the flow from the tank to each cylinder. Each cylinder then moves the appurtenant electrode assembly (aluminium arms, graphite electrodes, connecting cables, cooling water, etc.) in the vertical direction. The hydraulic actuators need to be capable of accelerating the electrode assembly $(\sim 17.000 \mathrm{~kg}$ for each electrode) to $\sim 100 \mathrm{~mm} / \mathrm{s}$ in about $100-500 \mathrm{~ms}^{21)}$ To maintain a constant pressure in the accumulating tank (64 bar), which has a capacity of $7 \mathrm{~m}^{3}$, three pumps, each capable of producing 230 bar of pressure and $0.14 \mathrm{~m}^{3} / \mathrm{min}$ of

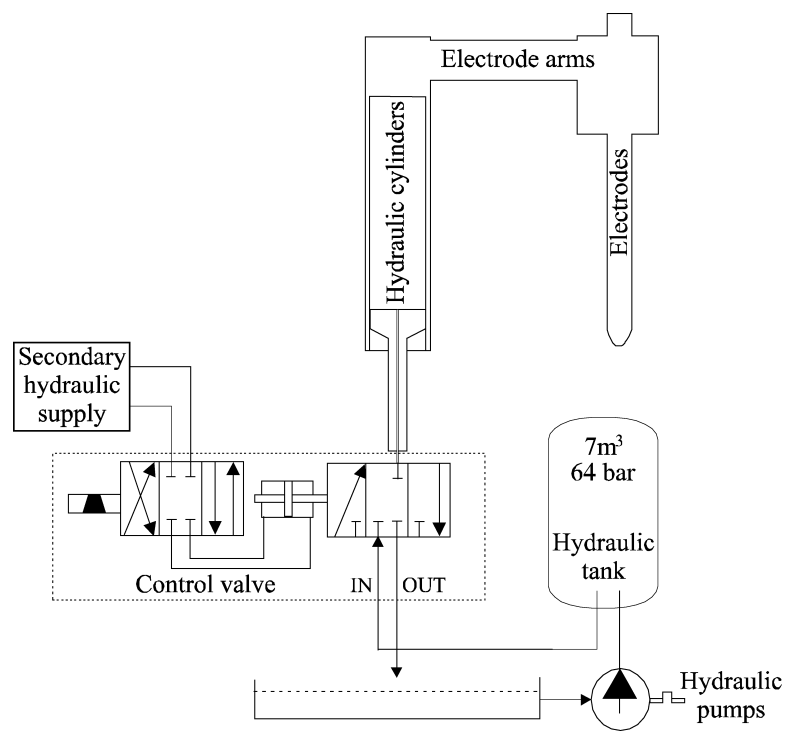

Fig. 3. Simplified schematic representation of the EAF hydraulic electrode positioning system. flow, are used. The pumps are engaged when the tank pressure drops below 60 bar and turn off when the pressure goes above 70 bar.

The dynamics of the hydraulic tank can be modeled by Eq. (21):

$$
\frac{d p_{s}}{d t}=\frac{E}{V_{t}} \cdot\left(q_{\text {in }}-q_{1}-q_{2}-q_{3}-q_{1 \_ \text {leak }}-q_{2 \_ \text {leak }}-q_{3 \_ \text {leak }}\right)
$$

where $p_{s}$ represents the tank pressure, $V_{t}$ represents the tank capacity $\left(7 \mathrm{~m}^{3}\right), q_{\text {in }}$ represents the fluid inflow into the tank $\left(0.42 \mathrm{~m}^{3} / \mathrm{min}\right), q_{i}(i=1, \cdots, 3)$ represents the fluid outflows from the tank (through the control valves to the electrode cylinders), $q_{i \text { leak }}(i=1, \cdots, 3)$ represents the leakage of the control valves and $E$ represents the bulk modulus of the hydraulic fluid, which can be obtained using Eq. (22) ${ }^{19)}$ :

$$
E=E_{l} \cdot \frac{1+\alpha_{G}\left(\frac{p_{a}}{p_{a}+p}\right)^{(1 / n)}}{1+E_{l} \alpha_{G} \frac{p_{a}^{(1 / n)}}{n\left(p_{a}+p\right)^{((n+1) / n)}}}
$$

where $E_{l}$ represents the modulus of the unmixed fluid, $p$ represents the pressure in the tank, $p_{a}$ represents the atmospheric pressure, $\alpha_{G}$ represents the relative gas content in the fluid at atmospheric pressure and $n$ represents the adiabatic index of the gas. For the needs of the hydraulic model we assume that the hydraulic suspension is not aerated and therefore has a bulk modulus of $E=1.8 \cdot 10^{9} \mathrm{~N} / \mathrm{m}^{2}$ ).

The electrode cylinders are operated via the EMG 7/422) control valves, which can be modeled using the flow and frequency-response characteristics shown in Fig. 4.

The inputs from the controller to the valve are within the interval of $[-10 \mathrm{~V}, 10 \mathrm{~V}]$ and lead to $[-100 \%, 100 \%]$ flow through the valve. The negative percentage of the valve openness means that the fluid is flowing from the cylinders back into the accumulator (through a sump), while the positive percentage of openness means that the fluid is flowing from the accumulator into the cylinders.

The control-valve dynamics can be described by Eq. $(23)^{19,23) \text { : }}$

$$
\begin{aligned}
& \text { If } l_{\text {in }}>0: \quad q_{i}=l_{\text {in }} \cdot K \cdot q_{\text {rated }} \sqrt{\left(p_{s}-p_{i}\right) / p_{\text {rated }}} \\
& \text { If } l_{\text {in }}<0: \quad q_{i}=l_{\text {in }} \cdot K \cdot q_{\text {rated }} \sqrt{\left(p_{i}-p_{a}\right) / p_{\text {rated }}}
\end{aligned}
$$

where $l_{\text {in }}$ represents the openness of the control valve
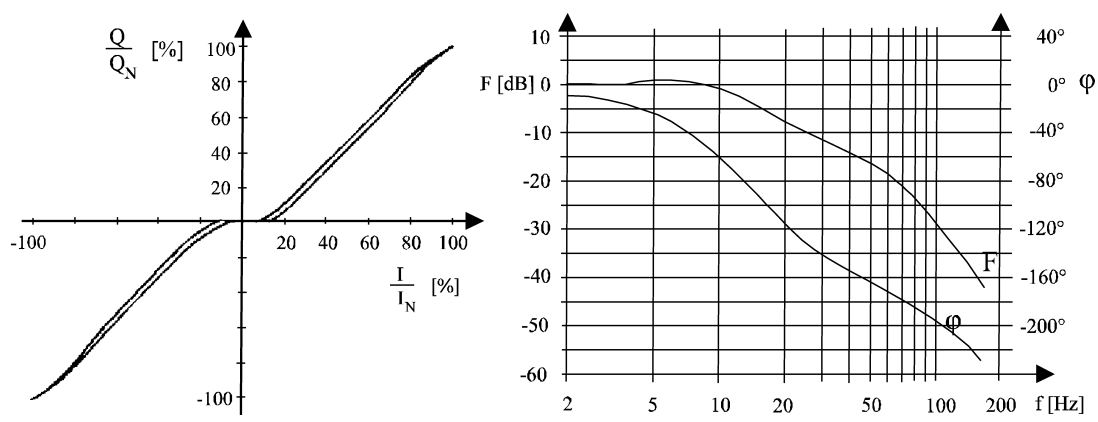

Fig. 4. Control valve characteristic: flow (left panel) and frequency response (right panel). 
[-100\%,100\%], $K\left(K=f\left(I / I_{N}\right)\right)$ represents a constant obtained from the flow characteristic graph in Fig. 4 (from a lookup table), $q_{\text {rated }}$ represents the rated flow of the control valve $\left(500 \mathrm{~d} \mathrm{~m}^{3} / \mathrm{min}\right), p_{\text {rated }}$ represents the rated pressure (60 bar), $p_{i}$ represents the pressure in the $i$-th cylinder, $p_{s}$ represents the supply pressure and $p_{a}$ represents the atmospheric pressure. As can be seen in Fig. 4 the control valve exhibits a hysteresis and the dynamics in the $[-20 \%, 20 \%]$ openness range are non-linear. (24):

The leakage of each valve can be defined by $C_{\text {leak }}$ in Eq.

$$
q_{i_{-} \text {leak }}=C_{\text {leak }} \cdot q_{\text {rated }}=0.5 \% \cdot q_{\text {rated }}
$$

The dynamics of the electrode cylinders, which are controlled by the control valves, can be modeled by the following equations:

$$
\begin{aligned}
& \frac{d x_{i}}{d t}=v_{i} \\
& F_{i}=p_{i} \cdot A \\
& \frac{d^{2} x_{i}}{d t^{2}}=-g+\frac{F_{i}}{m_{i}}-F_{\text {fric } \_i} \\
& \frac{d p_{i}}{d t}=\frac{E}{A \cdot\left|x_{i}\right|+V_{0}}\left(q_{i}-A v_{i}-q_{i_{-} \text {leak }}\right)
\end{aligned}
$$

where $i$ denotes the electrode $(1,2,3), x_{i}$ represents the electrode movement, $v_{i}$ represents the electrode speed, $F_{i}$ represents the hydraulic force, $p_{i}$ represents the pressure in the cylinder, $A$ represents the cross-sectional area of the rod $\left(\sim 0.28 \mathrm{~m}^{2}\right), g$ represents the standard gravity $\left(9.81 \mathrm{~m} / \mathrm{s}^{2}\right), m_{i}$ represents the electrode assembly weight $(\sim 17.000 \mathrm{~kg})$, $F_{\text {fric } \_i}$ represents the friction force, $q_{i}$ represents the incoming fluid flow, $q_{i \text { leak }}$ represents the valve leakage, $E$ represents the fluid bulk modulus and $V_{0}$ represents the dead-capacity of the cylinder. $F_{\text {fric } i i}$ combines the friction force, the drag and the Coulomb friction. ${ }^{20,24)}$

The control valves and electrode cylinders are controlled through a structure based on three uni-variable PID controllers. For each arc the controller computes the relative error between the reference and the actual resistance, and computes the relative valve openness [\%] through a given transfer function. The controller output is converted via an I/O card to voltage signals, which are the inputs to the control valve. The voltage signals are converted through an inverter to current signals, which then control the openness of the valve through a coil. Each valve contains a hydraulic pre-amplifier controlled by the current signals and the coil, which affect the dynamics of the primary hydraulic circuit actuating the electrode cylinders.

The electrode movements and consequently the arc lengths obtained from Eq. (25) are then used in Eq. (2) for computing the arc resistances, which are afterwards used by the controller to maintain the reference arc lengths. In this manner, the control feed-back loop for each phase is completed, which can be, together with the rest of the equations, schematically represented in Fig. 5, showing the block diagram of the developed EAF sub-models including the corresponding equation numbers and data flows (arrows).

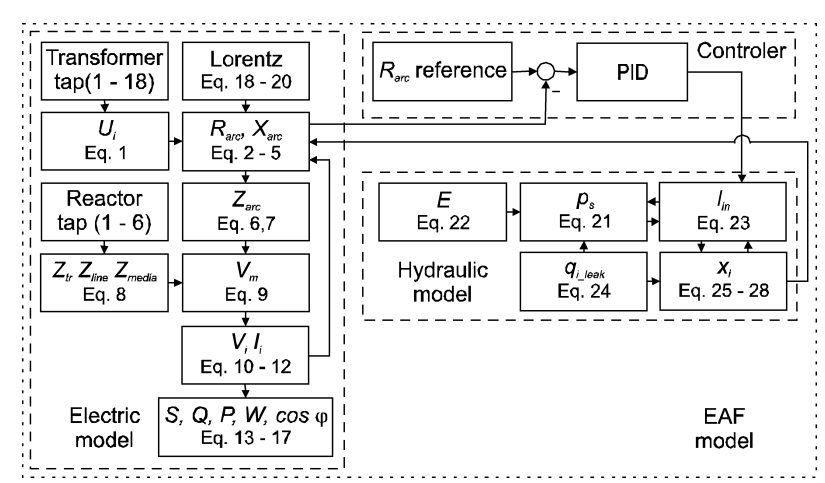

Fig. 5. Block diagram of the developed EAF sub-models including the corresponding equation numbers and data flows (arrows).

\section{Results}

The following section presents the simulation results obtained from the developed mathematical models for electrode control and a complete electric arc furnace in comparison with the measured EAF operational data. Since the EAF idle data (basket loading, electrode maintenance, etc.) does not represent any significant information about the melting process (except the tap-to-tap times, which are thus far not relevant for this study), it has been removed from the measurements and is therefore not used as a part of a model validation.

\subsection{Electrode Control Model}

The results of the electrode-control model were obtained by embedding the model in the EAF electric model. Figure 6 shows the results of the electrode-control model, where the inputs to the model are the controller outputs in $\%$, while the outputs of the model are the arc resistances in $\mathrm{m} \Omega$, the scrap height decrease and the arc length in $\mathrm{m}$. To verify whether the developed model and the controller are able to precisely track the reference signal, the simulation was performed without any additional noise (Lorentz attractor).

As is clear in Fig. 6 the electrode-control model is capable of arc reference tracking, which can also be seen at the times $100 \mathrm{~s}, 200 \mathrm{~s}$ and $300 \mathrm{~s}$. At $100 \mathrm{~s}$ the controller compensates the transformer tap change from tap 6 to tap 7 , which is visible from the longer arc length, which is needed to retain the reference resistance at the higher input voltage. At $200 \mathrm{~s}$ the controller compensates the arc reference change from 6 to $5 \mathrm{~m} \Omega$, which is visible from the shorter arc length, which is needed to decrease the arc resistance at the given input voltage. At $300 \mathrm{~s}$ arc 3 extinguishes, which is clear from the high resistance of arc 3 and the shorter lengths of the arcs 1 and 2 as each phase voltage is only $1 / 2$ of the phase-to-phase voltages, as already mentioned above. It is also clear that when the arc 3 extinguishes, the scrap height 3 stops decreasing since the third electrode has no thermal output.

\subsection{Electrical Model}

The subsequent section presents the simulation results obtained from the developed electrical model together with the electrode control in comparison to the measured EAF operational data. The model is validated for one heat (3 

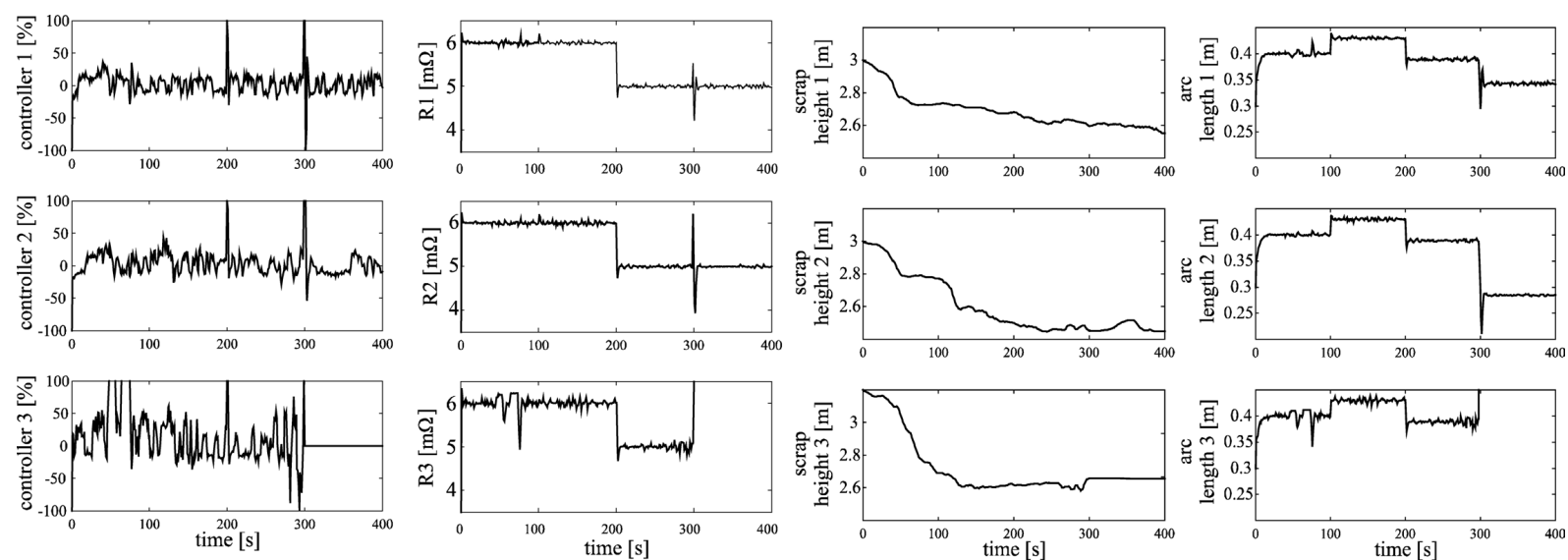

Fig. 6. Controller outputs in \% (1st column) and the corresponding arc resistances in $\mathrm{m} \Omega$ ( 2 nd column), the scrap decrease in $\mathrm{m}$ (3rd column) and the arc lengths in $\mathrm{m}$ (4th column).
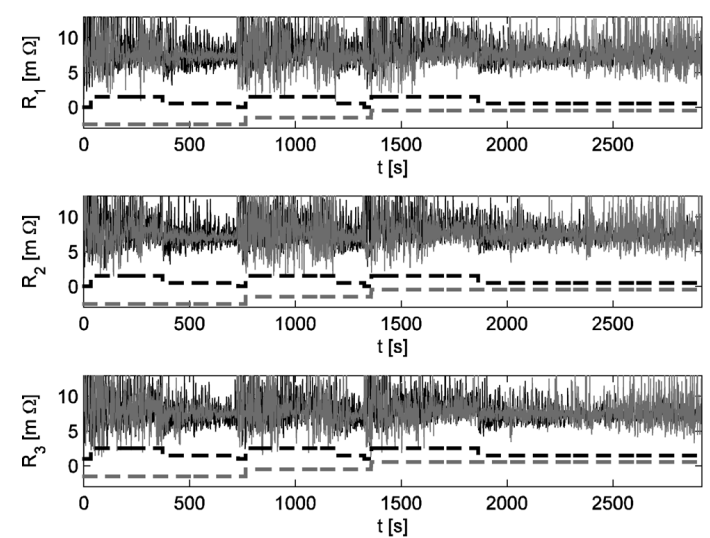
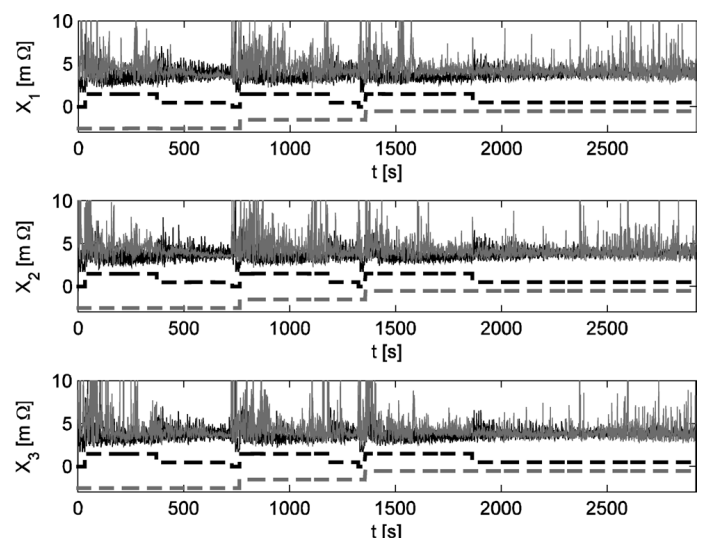

Fig. 7. Arc resistance (left panel) and reactance (right panel) in $\mathrm{m} \Omega$ for each phase; grey dashed line: current basket, black dashed line: current transformer tap, thin black line: simulated data, thin grey line: measured data.
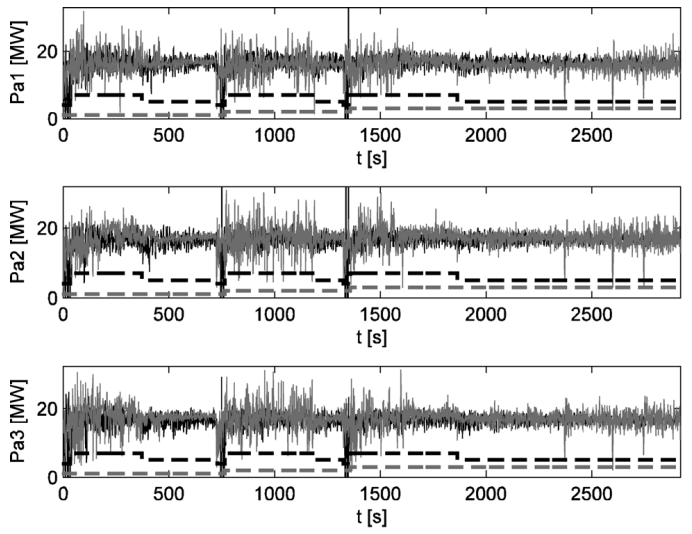
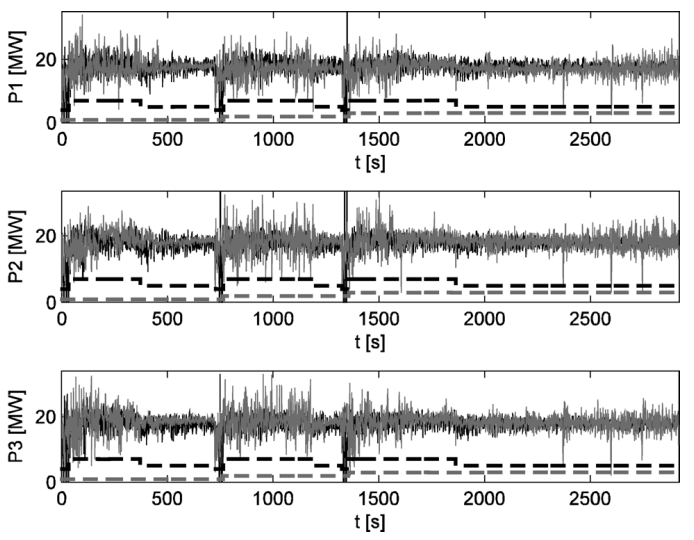

Fig. 8. Total arc power in MW (left panel) and total active power in MW (right panel) for each phase; grey dashed line: current basket, black dashed line: current transformer tap, thin black line: simulated data, thin grey line: measured data.

baskets of steel) with variable transformer taps (tap 6, 7, and 9) and fixed reactor tap (4). Figures $\mathbf{7}$ to $\mathbf{1 0}$ show the comparison between the measured and the simulated data for the arc resistances and reactances, the powers (apparent, active, arc and reactive) and the power factors for all three phases. Black and grey dashed lines are intended to denote the change in the selected transformer tap $(6,7,9)$ and in the progress of the basket loading $(1,2,3)$; however, their actual placement on each graph does not relate to the corresponding $y$-axis.
Figure 7 shows that the arc reactances increase each time when a new basket of scrap is loaded into the furnace and then exponentially decrease to a certain constant value. The reactance increase is a consequence of loading the solid steel, which increases the arc instability and its reactance. Furthermore, the arc-reactance decrease is a result of the steel melting and the slag formation. Also clear in Fig. 7 is that the behavior of the arcs is far more chaotic in the initial phases of melting when an additional basket of steel is loaded into the furnace, which is an indication of a lower 

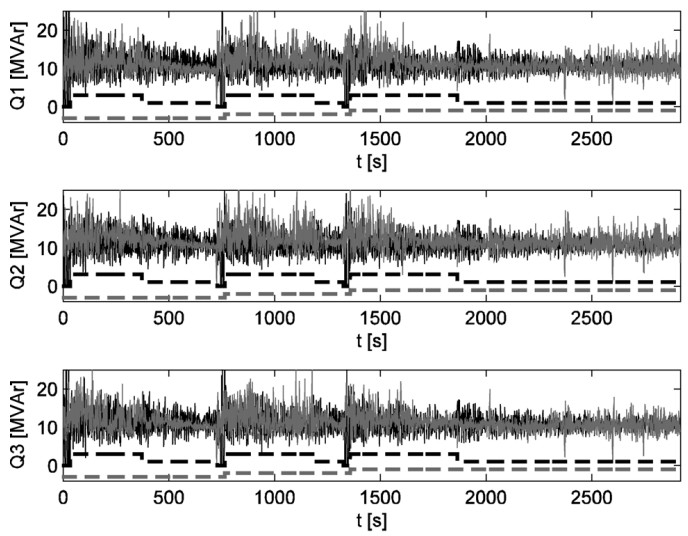
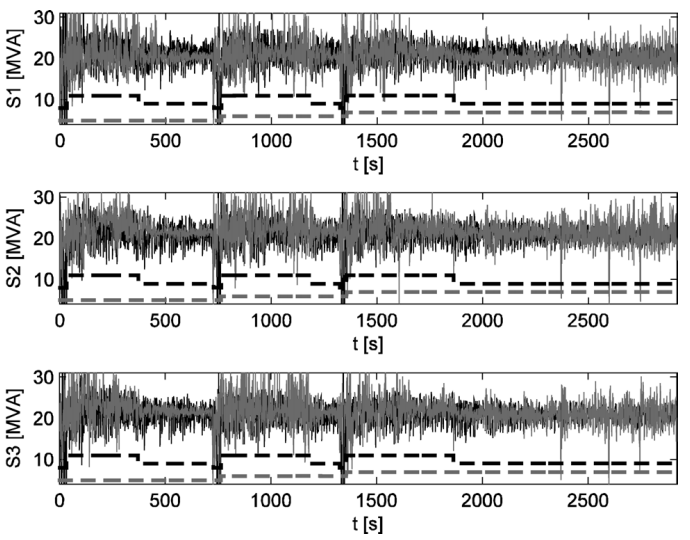

Fig. 9. Total reactive power in MVAr (left panel) and total apparent power in MVA (right panel) for each phase; grey dashed line: current basket, black dashed line: current transformer tap, thin black line: simulated data, thin grey line: measured data.

arc stability. From comparing the measured and simulated results it is clear that additional noise (chaos) contributes to a greater similarity of the compared signals and also proves the suitability of the developed model, supported by the presented equations.

Figures 8 and 9 show the results of the sub-models for simulating the active, arc, reactive and apparent powers. The computed data is similar to the obtained measurements. It can be seen that in the current setup the furnace consumes $\sim 18 \mathrm{MW}$ of active power per phase, $\sim 17.1 \mathrm{MW}$ arc power per phase, $\sim 11$ MVAr reactive power per phase and $\sim 21.5$ MVA apparent power per phase. The active and arc powers of the furnace could be increased to use the full apparent power potential of the furnace transformer (80 MVA); however, several furnace limitations and especially optimal power factors, which allow the maximum energy input, have to be taken into account when operating the EAF. Comparing the given values it is obvious that a great proportion of the consumed energy is lost due to the inner transformer and line impedances, the cathode voltage drops, the arc reactances and the additional inductive loads (furnace reactor), which are needed to stabilize the burning of the arcs, especially in the initial phases of the melting process, but add to the higher energy consumption and to the lower power factors (Fig. 10).

As is clear from Fig. 10 the EAF is operating with power factors of around $0.85-0.90$ due to the above-mentioned reactive loads, which worsen the active/reactive power ratio; however, they are needed because of the described drawbacks of the electric arcs.

Figure 11 shows the comparison between the measured and simulated total energy consumption.

As the aim of the developed EAF model is to use it for energy-consumption optimization, the simulated and measured consumptions of the electrical energy should be nearly identical. The deviations between the measured and simulated data, which can be observed in Fig. 11, are minimal, which indicates the appropriateness of the developed model for the above-mentioned aims. Also, by recalculating the total energy consumption for a ton of molten steel (furnace capacity $\sim 90$ tons) it is clear that the furnace in the current setup uses $\sim 500 \mathrm{kWh}$ /ton of steel, which should be further lowered by applying different kinds of optimization procedures.

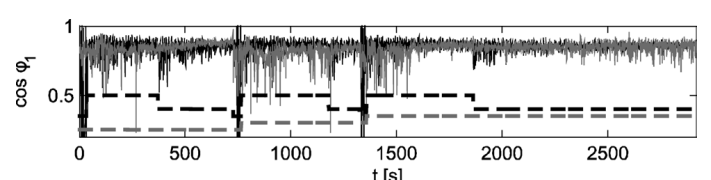

$\mathrm{t}[\mathrm{s}]$
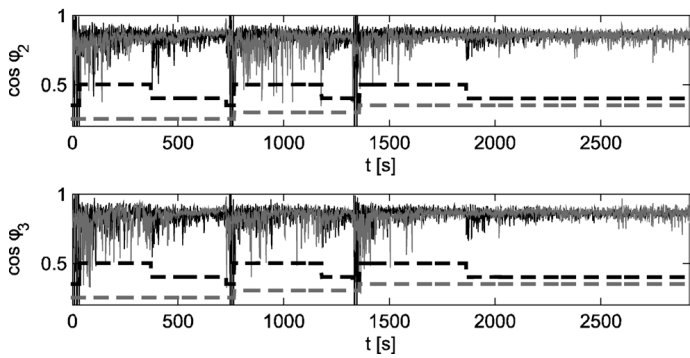

Fig. 10. Power factor for each phase; grey dashed line: current basket, black dashed line: current transformer tap, thin black line: simulated data, thin grey line: measured data.

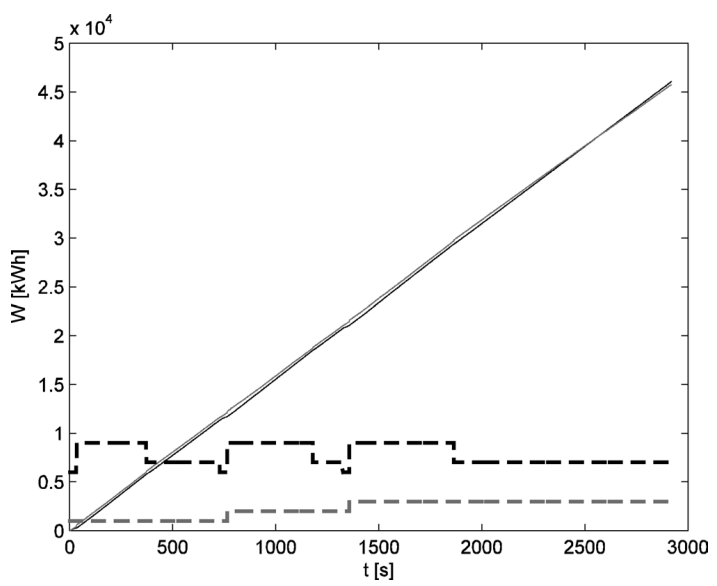

Fig. 11. Total energy consumption in $\mathrm{kW} h$ per heat; grey dashed line: current basket, black dashed line: current transformer tap, thin black line: simulated data, thin grey line: measured data.

As shown by Figs. 7 to 11 all the measured and simulated EAF data demonstrate high levels of similarity, which proves the accurate modeling of the EAF's behavior in different operational situations. In this manner, the validation of the presented model can be considered as successful. Moreover, the chaotic conditions added by the Lorentz attractor contribute to the greater realism of the model's outputs, when compared to the real-time EAF measurements. 

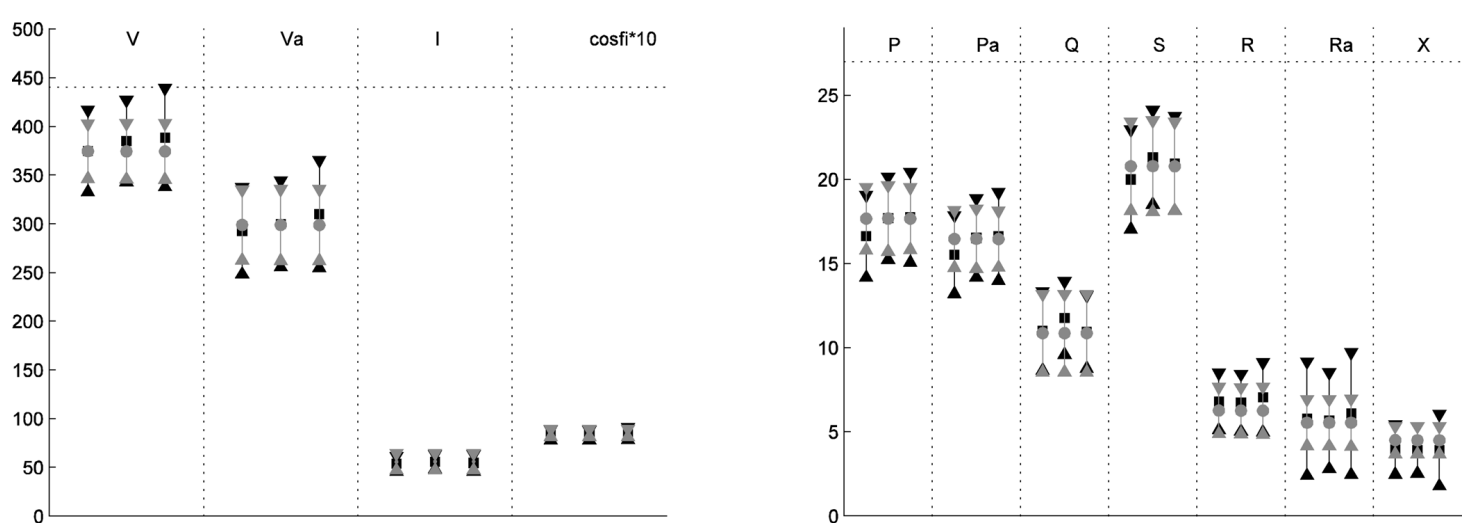

Fig. 12. Average measured and simulated values including the standard deviations; $V$ : secondary transformer voltages $[\mathrm{V}], V_{a}$ : arc voltages [V], I: phase currents [kA], $\cos \varphi$ : power factors $(* 10), S$ : total apparent powers [MVA], $P$ : active powers [MW], $P_{a}$ : arc powers [MW], $Q$ : reactive powers [MVAr], $R$ : total circuit resistances $\left[\mathrm{m} \Omega\right.$ ], $R_{a}$ : $\operatorname{arc}$ resistances $[\mathrm{m} \Omega], X$ : total circuit reactances $[\mathrm{m} \Omega]$; black squares: measured average value, grey circles: simulated average value, black triangles: measured standard deviations, grey triangles: simulated standard deviations.
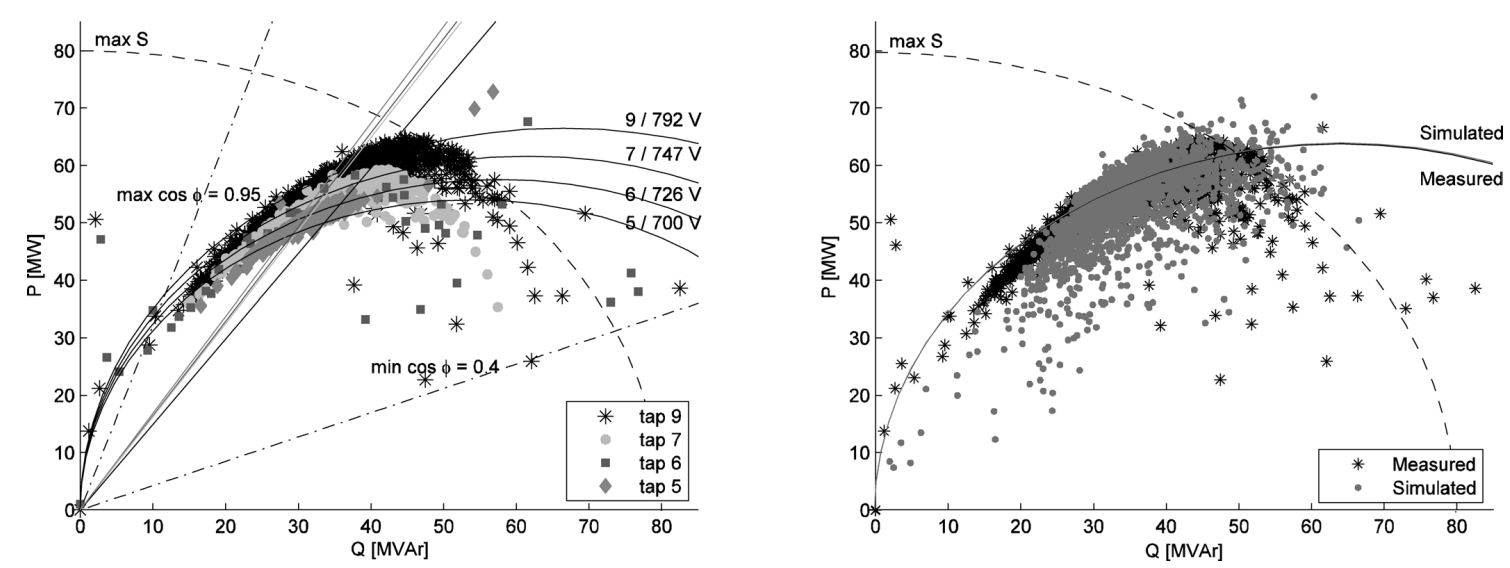

Fig. 13. Left panel: Power locus diagram of the simulated values for transformer taps $5,6,7$ and $9 ; 5 / 700$ to $9 / 792 \mathrm{~V}$ : ideal EAF power curves for the simulated taps, the diagonal lines represent the average constant power-factor values $(0.86,0.855,0.85$ and 0.83 ) for the selected taps in a ascending order from left (tap 5) to right (tap 9); Right panel: Power locus diagram of the simulated values in comparison to the measured values; simulated and measured power curves are drawn (average power for all transformer taps combined); however, the difference between them is minimal.

To support the last statement, Fig. 12 shows the average measured and simulated RMS values, including the standard deviations of the signals for one heat (the same data as in Figs. 7 to 11) of the EAF melting process.

As Fig. 12 shows, the average values and standard deviations of the data computed by the model correspond to the obtained measurements, which further proves the similarity between the developed model and the actual EAF melting process.

An another important aspect of the quality of the melting-process modeling is the power locus diagram or the operational diagram of the EAF. Figure 13 shows the $P$ vs. $Q$ diagram of the simulated values for four operational transformer taps $(5,6,7$ and 9 with the secondary transformer voltages $700 \mathrm{~V}, 726 \mathrm{~V}, 747 \mathrm{~V}$ and $792 \mathrm{~V})$. The circles represent the ideal power locus curves, the diameters of which are proportional to the maximum furnace power for the selected transformer tap.

As is clear from Fig. 13 (left panel) the values for the reactive $(Q)$ and active $(P)$ powers computed by the developed model lie rather close to the range of the ideal power curves. Furthermore, it is clear that the majority of the sim- ulated values are placed within the maximum apparent power $(\max S$ ) curve, which also matches the measured data. The power factor lines placed from the left to the right correspond to the transformer taps in ascending order (5-9). A few of the simulated results lie in the area that leads to EAF operation with the maximum and minimum achievable power factors; however, the maintenance of such values is only possible for very short times, as operating with the maximum power factor causes severe arc-instability problems, while operating with a minimum power factor requires very short arcs with a minimal distance between the electrodes and the scrap metal, which represents a risk of slag and scrap carbonization. Also clear in Fig. 13 (right panel), the majority of the simulated and the measured $P v s$. $Q$ values are positioned in the operational area of the EAF, which further supports the results presented in the other figures and the appropriateness of the developed model for the case of a real EAF melting process.

\section{Conclusion}

In this paper an approach to the mathematical modeling 
of electrical EAF processes and hydraulic electrode control is presented. Regarding the presented results, the developed model can be considered as appropriate for the initial aims of the study, since high levels of similarity between the modeled and the measured EAF data can be achieved. This also proves the suitability of the proposed model for further development and analysis (simulator, control-design purposes and energy optimization). The presented work thus far represents the first step in a complete EAF model, which will be further complemented by thermal, chemical and energy-balance sub-models, in order to use its full potential. Nonetheless, developing an accurate and reliable electric model with electrode control is crucial, because most of the other sub-models are directly dependent on the characteristics of the model presented in this paper.

\section{REFERENCES}

1) K. J. Tseng, Y. Wang and D. M. Vilathgamuwa: IEEE Trans. Power Electron., 12 (1997), 429.

2) Y. Lee, H. Nordborg, Y. Suh and P. Steimer: 22nd Annu. IEEE Appl. Power Electron. Conf., IEEE, New Jersey, USA, (2007), 1280.

3) M. P. A. Anxo and M. D. Pérez: IEEE Trans. Power Delivery, 19 (2004), 367.

4) R. Collantes-Bellido and T. Gómez: IEEE Trans. Power Delivery, 12 (1997), 1812.

5) H. Mokhtari and M. Hejri: Transm. and Distrib. Conf., IEEE/PES 3 , Vol. 3, IEEE, New Jersey, USA, (2002), 2078.

6) G. C. Montanari, M. Loggini, A. Cavallini, L. Pitti and D. Zaninelli: IEEE Trans. Power Delivery, 9 (1994), 2026.

7) T. Zheng and E. B. Makram: IEEE Trans. Power Delivery, 15
(2000), 931.

8) Spie-Trindel, France: Electrode Regulation-E.M.P.E.R.E, SpieTrindel, Uckange, France, (2001), 1.

9) A. M. Cassie: Int. Conf. on Large Electric High-Tension Systems, C.I.G.R.E., Paris, France, (1939), 1.

10) O. Mayr: Archiv für Elektrotechnik, 37 (1943), 588.

11) S. Köhle: 10. UIE Kongress Stockholm, Sweden, (1984).

12) B. Bowman: Metall. Int., 4 (1988), 286.

13) S. Köhle, M. Knoop and R. Lichterbeck: Elektrowärme Int., 51 (1993), 175.

14) A. Z. Nemirovskii and F. Puchkarev: J. Phys. D: Appl. Phys., 25 (1992), 798.

15) J. Bratina: Elektroobločna peč, Slovenske Železarne, Ravne na Koroškem, (1994), 10.

16) E. Kirchenmayer: Electrical Engineering of Arc Furnaces-Furnace Transformers, Institut für Bildung im Stahl-Zentrum, Hamburg, (2003), 8 .

17) G. Jang, W. Wang, G. T. Heydt, S. S. Venkata and B. Lee: Electr. Power Compon. Syst., 29 (2001), 1060.

18) R. D. M. MacRosty and C. L. E. Swartz: AIChE J., 53 (2007), 640

19) H. Dörr, R. Ewald, J. Hutter, D. Kretz, F. Liedhegener and A. Schmitt: Der Hydraulik Trainer Band 2, Mannesmann Rexroth GmbH, Lohr am Main, (1986).

20) B. Yao, F. Bu, J. Reedy and G. T. C. Chiu: IEEE/ASME Trans. Mechatron., 5 (2000), 77.

21) G. Schaefers: Electrical Engineering of Arc Furnaces-Electrode Control of Arc Furnaces, Institut für Bildung im Stahl-Zentrum, Hamburg, (2003), 3.

22) Elektro-Mechanik GmbH, Germany: EMG Manual-Moving Coil Regulators TR 7/3, 8/3, 7/4, Elektro-Mechanik GmbH, Wendenerhütte, Germany, (1985).

23) Fisher Controls International, U.S.A: Control Valve Handbook, Fisher Controls International, Marshalltown, U.S.A., 4 (2005), 114.

24) A. Alleyne and R. Liu: Control Eng. Pract., 8 (2000), 1347. 